\title{
Effect of Patient Centered Care on Management of Childhood Bronchial Asthma: An Educational Intervention Study
}

\author{
${ }^{1}$ Amany M. Abdallah, ${ }^{2}$ Hosnia M. Ragab, ${ }^{3}$ Soad A. Shedeed and ${ }^{2}$ Ghada M. Khalil \\ ${ }^{1}$ Family Medicine Department, Faculty of Medicine, ${ }^{2}$ Community, Environmental and \\ Occupational Medicine Department, ${ }^{3}$ Pediatric Department Zagazig University, Egypt.
}

\begin{abstract}
Objective: to assess medication adherence and quality of life (QoL) in children with asthma, to plan and implement an intervention to evaluate the outcome of patient centered care. Method: An interventional study was conducted on 82 children with asthma attending pediatric outpatient chest clinic at Zagazig University Hospitals. They were assigned into experimental or control groups randomly (41 patients in each). All patients, at first visit and after three months underwent assessment of medication adherence, inhaler technique and QoL, determination of asthma symptoms control and measuring pulmonary function. Experimental group only was subjected to face-to-face tailored patient education for about thirty minutes and follow-up visits every two weeks. Control group was subjected to routine clinical consultation Results: There was a highly significant improvement in all assessed variables among intervention group when compared with control group including QoL. The Experimental group had high medication adherence, accurate inhaler technique and, which were associated with well-controlled asthma. Conclusion: patient centered care approach to children with asthma and their caregivers could result in improving asthma outcomes in terms of medication adherence, asthma control and QoL.
\end{abstract}

Key words: asthma, patient centered, quality, adherence.

Corresponding author: Amany M. Abdallah. $\quad$ E-mail:dr.amanymohammed@gmail.com

\section{Introduction}

Bronchial asthma is a chronic disease which is characterized by variability of its respiratory symptoms in frequency and intensity, with variable expiratory airflow restriction, chronic air passages inflammation is usually considered. ${ }^{1}$ It affects all ages but usually occurs early in childhood and it is the most common respiratory disease among children , affecting $10 \%$ to $15 \%$ of them. ${ }^{2}$ Asthma is a major cause of pediatric hospital admissions and emergency department (ED) visits. ${ }^{3}$ Its prevalence among Egyptian children was $7.7 \%$ as demonstrated by study used questionnaire diagnosed asthma. $^{4}$
Person-centered care is not just about giving patients whatever they want or providing information. It is considering patient's desires, values, family situations, social circumstances and lifestyles; seeing the person as an individual, and working together to plan, deliver and evaluate health service. $^{5}$

Patient education is one of the pillars for proper asthma management. ${ }^{6}$ People living with asthma have to accommodate their long-term condition within the context of their daily life. They may need to remember to use regular medication, to keep a supply of inhalers, avoid their triggers where possible, cope with the variability of asthma 
Table (1): Comparison between the two studied groups regarding gender and age:

\begin{tabular}{|c|c|c|c|c|c|c|}
\hline \multirow{2}{*}{ Medication adherence } & \multicolumn{2}{|c|}{$\begin{array}{c}\text { Experimental } \\
\text { group }\end{array}$} & \multicolumn{2}{|c|}{$\begin{array}{c}\text { Control } \\
\text { group }\end{array}$} & \multirow[t]{2}{*}{$X^{2}$} & \multirow[t]{2}{*}{$\mathbf{P}$} \\
\hline & $\mathbf{N}$ & $\%$ & $\mathbf{N}$ & $\%$ & & \\
\hline $\begin{array}{l}\text { Gender: } \\
\text { - } \quad \text { Male } \\
\text { - female }\end{array}$ & $\begin{array}{l}20 \\
21\end{array}$ & $\begin{array}{l}48.8 \\
51.2\end{array}$ & $\begin{array}{l}17 \\
24\end{array}$ & $\begin{array}{l}41.5 \\
58.5\end{array}$ & 0.44 & $0.51(\mathrm{NS})$ \\
\hline $\begin{array}{l}\text { Age group } \\
\text { - } \quad<7 \text { years old } \\
\text { - } \quad \geq 7 \text { years old }\end{array}$ & $\begin{array}{l}14 \\
27\end{array}$ & $\begin{array}{l}34.1 \\
65.9\end{array}$ & $\begin{array}{l}22 \\
19\end{array}$ & $\begin{array}{l}53.7 \\
46.3\end{array}$ & 3.17 & $0.08(\mathrm{NS})$ \\
\hline
\end{tabular}

NS statistically non-significant

and the impact they have on their and their family's lifestyle. They have to recognize when their asthma is deteriorating and make decisions about when to adjust their medication, when to use emergency treatment and when to seek professional help. ${ }^{7}$

The main objective of this study is to assess medication adherence and QoL of children with asthma, to plan and implement patient centered care approach on them and to evaluate the outcome of implementation of patient centered care.

\section{Method}

Study setting and design: This interventional study was conducted during the period from December 2017 to May 2018 in pediatric pulmonology outpatient clinic at Zagazig University hospitals, which was selected due to high attendance rate of children with asthma and better quality of care on hands of professional physicians with availability of getting repeated follow-up visits.

Study population and sample size: Assuming that knowledge about inhalation technique has changed from $15.4 \%$ to $46.2 \%$ in asthmatic patients after patient education ${ }^{(8)}$, sample size was calculated to be 82 asthmatic children; half of them as intervention group and the other half as control group, using EPI INFO at power $80 \%$ and C.I $95 \%$.
Inclusion criteria: Children with asthma (from 1 to 18 years old) attend pediatric pulmonology outpatient clinic at Zagazig university hospitals regardless of their asthma condition.

Exclusion criteria: Patients having other lung co-morbidities, including congenital (e.g., cystic fibrosis) and airway infections; patient with complication including; heart failure (cor-pulmonale) or associated with congenital heart disease.

Sampling technique: A systematic random sampling technique was adopted for selection of cases. As the average attendance of asthmatic children is 21 children/day and sample size of three children/day was needed, so each seventh child starting from random number selected out of first seven numbers was chosen in the sample. Because asthmatic children might attend for follow up visits, if the child was repeated, the next child would be chosen instead of him or her. Cases were alternatively divided into two equal groups (intervention and control).

Study tools: (1) Eight items morisky medication adherence scale (MMAS-8): This scale consists of eight questions, first seven items having a dichotomous answer (yes/no) where yes $=1$ indicating non adherent behavior and no $=0$ indicating adherent behavior. For item 8 , a patient or his family can choose an answer on 5 points likert scale, expressing how often happens that a patient does not take his medications. Cut-off values for categorizing patients as having a high, medium or low. ${ }^{9}$ The 
Table (2): Comparison between medication adherence, level of asthma symptom control and accuracy of inhaler technique of the two studied groups before and after intervention:

\begin{tabular}{|c|c|c|c|c|c|c|}
\hline \multirow{2}{*}{ Medication adherence } & \multicolumn{2}{|c|}{ Experimental group } & \multicolumn{2}{|c|}{ Control group } & \multirow{2}{*}{$X^{2}$} & \multirow{2}{*}{$\mathbf{P}$} \\
\hline & $\mathbf{N}$ & $\%$ & $\mathbf{N}$ & $\%$ & & \\
\hline \multicolumn{7}{|l|}{ Before intervention: } \\
\hline$\overline{\text { - High }}$ & 1 & 2.4 & 2 & 4.9 & & \\
\hline - Medium & 6 & 14.6 & 8 & 19.5 & 0.76 & $0.7(\mathrm{NS})$ \\
\hline - Low & 34 & 82.9 & 31 & 75.6 & & \\
\hline \multicolumn{7}{|l|}{ After intervention: } \\
\hline - High & $21^{\infty}$ & 51.2 & 2 & 4.9 & & \\
\hline - Medium & 15 & 36.6 & 10 & 24.4 & 33.6 & $<0.001(\mathrm{HS})$ \\
\hline - Low & 5 & 12.2 & 29 & 70.7 & & \\
\hline $\mathbf{P}(\mathrm{McNemar}$ test $)$ & \multicolumn{2}{|c|}{$<0.001$ (HS) } & \multicolumn{2}{|c|}{$0.32(\mathrm{NS})$} & & \\
\hline \multicolumn{7}{|l|}{ Before intervention: } \\
\hline - well controlled & 1 & 2.4 & 1 & 2.4 & & \\
\hline - Partially controlled & 15 & 36.6 & 13 & 31.7 & 0.22 & $0.89(\mathrm{NS})$ \\
\hline - Un controlled & 25 & 61 & 27 & 65.9 & & \\
\hline \multicolumn{7}{|l|}{ After intervention: } \\
\hline - Well controlled & 27 & 65.9 & 9 & 22 & & \\
\hline - Partially controlled & 12 & 29.3 & 14 & 34.1 & 21.95 & $<0.001(\mathrm{HS})$ \\
\hline - Un controlled & 2 & 4.9 & 18 & 43.9 & & \\
\hline $\mathbf{P}(\mathrm{McNemar}$ test $)$ & \multicolumn{2}{|c|}{$<0.001(\mathrm{HS})$} & \multicolumn{2}{|c|}{$0.03(\mathrm{~S})$} & & \\
\hline \multicolumn{7}{|l|}{ Inhaler technique } \\
\hline \multicolumn{7}{|l|}{ Before intervention: } \\
\hline - accurate & 8 & 19.5 & 4 & 9.8 & & \\
\hline - inaccurate & 33 & 80.5 & 37 & 90.2 & Fisher & $0.2(\mathrm{NS})$ \\
\hline \multicolumn{7}{|l|}{ After intervention: } \\
\hline - accurate & 27 & 65.9 & 14 & 34.1 & $\left(x^{2}\right)$ & $0.004(\mathrm{~S})$ \\
\hline - inaccurate & 14 & 34.1 & 27 & 65.9 & 8.2 & \\
\hline $\mathbf{P}$ (McNemar test) & \multicolumn{2}{|c|}{$0.023(\mathrm{~S})$} & \multicolumn{2}{|c|}{$0.003(\mathrm{~S})$} & & \\
\hline
\end{tabular}

S statistically significant HS statistically highly significant NS statistically non-significant

MMAS-8 is valid for assessing medication adherence in patients with asthma. $^{10}$ Validation of the questionnaire was made as follows: the questionnaire was translated using a back-translation technique. (2) Pediatric asthma quality of life questionnaire (PAQLQ) has been validated for use among children between the ages of 7 and 17 years, it included 23 items in three domains (5 questions of activity limitation domain, 10 questions of symptoms domain and 8 questions emotional function domain)..$^{11}$ (3) Pediatric asthma caregiver's quality of life questionnaire (PACQLQ) was used for guardian of children with asthma between
1-7 years it included 13 items. Four items concern activity limitation and nine concern emotional function. Responses to each item in the PACQLQ are given on a 7-point likert scale where 1 represents severe impairment and 7 represents no impairment. The questionnaire asks caregivers to recall impairments experienced during the previous week. ${ }^{12}$ (4) Checklist for inhaler technique: The patients were asked to show how they use their inhaler and check their technique against the checklist for that type most commonly used inhalers are pressurized metered dose inhalers with spacer and dry powder inhalers. Optimal technique was 
Table (3): Percentage of change of lung function parameters of studied groups:

\begin{tabular}{lcccc}
\hline Percentage of change & Experimental group & Control group & Z & P \\
\hline FEV $_{1}:$ Mean \pm SD & 30.1 & 3.7 & & \\
Range & $4.5-65.4$ & $5.1-12.4$ & -5.58 & $<0.001(\mathrm{HS})$ \\
\hline PEF: Median & 56.9 & 18.6 & & \\
Range & $18.7-121.7$ & $1.2-44.9$ & -3.25 & $0.001(\mathrm{HS})$ \\
\hline
\end{tabular}

$S$ statistically significant $H S$ statistically highly significant $N S$ statistically non-significant Z Mann Whitney test Forced Expiratory Volume in the first second ( $\left.F E V_{l}\right)$, Peak Expiratory Flow (PEF)

defined as successful execution of each step on the checklist without errors. ${ }^{13}$ (5) GINA guidelines for determining the level of asthma symptoms control: To assess symptoms control the following was asked about over the past four weeks: frequency of daytime and nighttime asthma symptoms, reliever use for relief of symptoms and activity limitation. Levels of asthma related symptoms were classified as well controlled if none of these are present, partly controlled if 1 or 2 of these are present and uncontrolled if 3 or 4 of these are present .Children who are five years or younger varies within each category. ${ }^{14}(6)$ Pulmonary function test (PFT): Pulmonary function was assessed with the use of digital spirometer (Viasys Health care GmbH leibnizstrasse D-97204 Hoechberg, Germany) at the initial interview and three months later.

Forced Vital Capacity (FVC), Forced Expiratory Volume in the first second $\left(\mathrm{FEV}_{1}\right)$, ratio of $\mathrm{FEV}_{1} / \mathrm{FVC}$, and Peak Expiratory Flow (PEF) were recorded. All parameters are expressed as a percentage of predicted values for age, gender, and height. ${ }^{15}$ Results of PFT are not reliable in patients younger than five years who are unable to perform the conventional spirometry maneuver, so children $\geq 6$ years only had done PFT. In asthma, lung function may vary between completely normal and severely obstructed in the same patient. Poorly controlled asthma is associated with greater variability in lung function than well controlled asthma. ${ }^{14}$
Operational design

Pilot study: Before starting to collect the data, a pilot study was conducted on $10 \%$ of the sample size to test the feasibility of the study, as well as the clarity of the tools and to estimate the time needed to fill each questionnaire. The pilot study findings showed that the interviewing questionnaires were clear and relevant. No modification was done.

Field work: First visit: (A) For control and Experimental groups: the following data was collected; personal history, history of present illness, other diseases or associated co-morbidities, past history of daily activities, frequency of daytime symptoms and nocturnal awakening, number and forms of medication used, regularity of asthma medication use, inhalation technique and Family history of asthma or atopy. Clinical examination was done for each child to exclude asthma exacerbation at time of interview. Assessment of socioeconomic status by ElGilany et.al ${ }^{16}$ questionnaire and filling pretest questionnaires (MMAS-8 and PAQLQ or PACQLQ) for each patient with his or her caregiver. Each questionnaire took about 15 minutes to be filled. Determining level of asthma symptom control. Checking correctness of inhaler technique. PFT was agreed to be done in children $\geq 6$ years. (B) Tailored patient education for each patient and his or her caregiver (for experimental group only): The educational sessions, each lasted for about 40 minutes, were conducted at the 
Table (4): Comparison of pretest and posttest pediatric asthma quality of life questionnaire scores of studied participants $\geq 7$ years:

\begin{tabular}{|c|c|c|c|c|c|c|}
\hline \multirow{2}{*}{ Variables } & \multicolumn{2}{|c|}{$\begin{array}{l}\text { Experimental group } \\
\qquad(n=27)\end{array}$} & \multicolumn{2}{|c|}{$\begin{array}{c}\text { Control group } \\
(n=19)\end{array}$} & \multirow[t]{2}{*}{ Test } & \multirow[t]{2}{*}{$\mathbf{P}$} \\
\hline & Mean \pm SD & Range & Mean \pm SD & Range & & \\
\hline $\begin{array}{l}\text { Activity limitation } \\
\text { domain: }\end{array}$ & & & & & & \\
\hline Pre test & $2.8 \pm 1.1$ & $1-3$ & $2.1 \pm 0.87$ & $1-3$ & (Z) -1.5 & 0.1 (NS) \\
\hline Post test & $5.6 \pm 1.1$ & $2.2-6.8$ & $1.8 \pm 0.55$ & $1-2.4$ & (t) 14.2 & $<0.001(\mathrm{HS})$ \\
\hline P value & \multicolumn{2}{|c|}{$<0.001(\mathrm{HS})$} & \multicolumn{2}{|c|}{$0.03(\mathrm{~S})$} & & \\
\hline
\end{tabular}

\section{Symptoms}

domain:

Pre test

$$
2.8 \pm 1.1 \quad 1.3-4.4
$$

$2.9 \pm 1.2$

$1.1-4$

(Z) -0.3

0.8 (NS)

Post test

$5.5 \pm 0.9 \quad 3-6.6$

$2.8 \pm 0.94$

$1.8-4.2$

(t) 9.9

$<0.001$ (HS)

$P$ value $<0.001(\mathrm{HS})$ 0.7 (NS)

Emotions domain
Pre test

Post test

$$
2.6 \pm 0.88 \quad 1-4.4 \quad 2.6 \pm 0.52
$$

$$
1.75-3
$$

(Z) -0.06

0.9 (NS)

$P$ value

$$
5.7 \pm 1.2 \quad 2.4-7
$$

$2.5 \pm 0.79$

$1.5-3.7$

(t) 10.3

$<0.001$ (HS)

\section{Total PAQLQ:}

Pre test

Post test $<0.001$ (HS) 0.5 (NS)

$P$ value

$$
2.7 \pm 0.95 \quad 1.1-4.04
$$

$2.6 \pm 0.8$

$1.3-3.2$

(Z) -1.4

0.1 (NS)

$5.6 \pm 0.96 \quad 2.6-6.4$

$2.5 \pm 0.77 \quad 1.5-3.6$

(t) 11.7 $<0.001$ (HS)

S statistically significant HS statistically highly significant NS statistically non-significant Z Mann Whitney test t independent sample t test $P A Q L Q$ pediatric asthma quality of life questionnaire

pediatric chest outpatient clinic at Zagazig University Hospitals The educational sessions were held in the form of personal interview and one to one discussion $u$, included information about: basic knowledge about bronchial asthma (definition, causes including triggers, symptoms, preventive measures, advantage of inhaler use), what define well-controlled bronchial asthma and the patient's current level of control, role of medication, importance of asthma action plan that help patient to quickly recognize and cope with early warning signs of an asthma attack, the appropriate use of metered dose inhaler with spacer or dry powder inhaler according to need, and regular review by a clinician of the status of the patient's bronchial asthma control. A printed material was given to each patients including all information thy received in the personal interview. For experimental group only, follow up visits were held every two weeks for three months through personal interview or telephone calls during these visits we revise the message, and inhaler technique.

Last visit: After three months from the first visit for experimental group and control group. (1) Filling the posttest questionnaires. (2) Reassessment of level of asthma symptoms control. (3) Rechecking the inhaler technique. (4) Repeating PFT for those $\geq 6$ years.

\begin{tabular}{|c|c|c|c|c|c|c|}
\hline \multirow{2}{*}{ 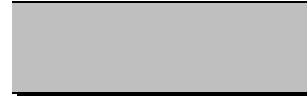 } & \multicolumn{2}{|c|}{ Experimental group } & \multicolumn{2}{|c|}{ Control group } & \multirow{2}{*}{ Test } & \multirow[b]{2}{*}{$\mathbf{P}$} \\
\hline & Mean \pm SD & Range & Mean \pm SD & Range & & \\
\hline
\end{tabular}

Administrative and ethical design and approval: The study protocol was approved from the ethical committee at Faculty of Medicine Zagazig University and IRB "institutional review board". An

Table (5): Comparison of pretest and posttest pediatric asthma caregiver's quality of life questionnaire scores of the guardian of studied participants between 1-7 years: 


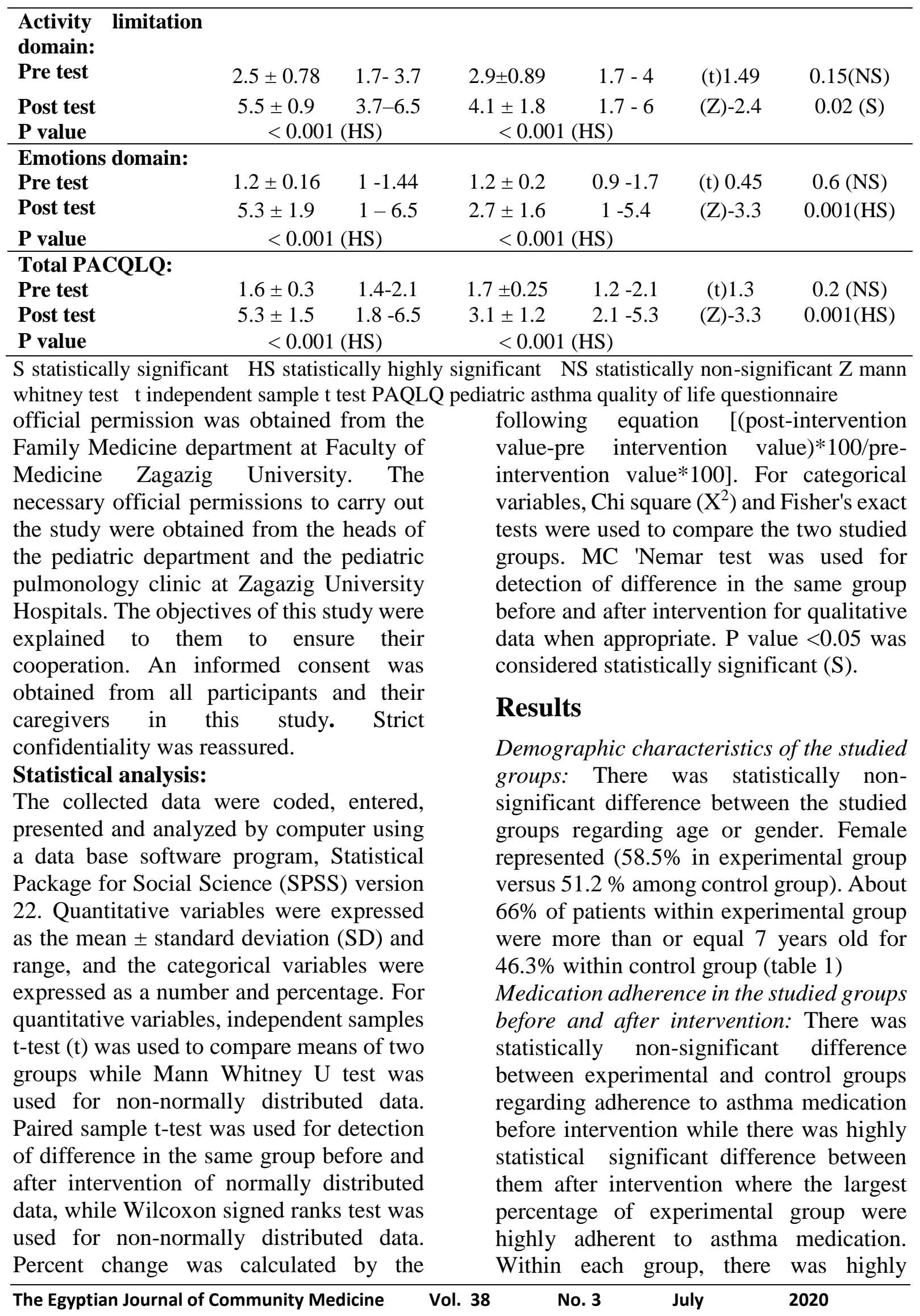


statistical significant improvement among experimental group after intervention while there was no statistically significant improvement among control group (table 2).

Level of asthma symptom control and accuracy of inhaler technique: There was statistically non-significant difference between experimental and control groups regarding level of asthma symptom control or accuracy of inhaler technique before intervention while there was highly statistical significant difference between them after intervention where the largest percentage of experimental group had their symptoms well controlled to asthma medication and use their inhalers accurately. Within each group, there was statistical significant improvement over time (table 2).

Pulmonary function test in the studied groups before and after intervention: There was highly statistically significant improvement among experimental group when compared with control group regarding percentage of change of values of lung function parameters (table 3 ).

QoL in in the studied groups before and after intervention: In either patient reported (PAQLQ) or caregivers' reported one, there were no statistically significant differences between experimental group and control group when comparable regarding all subcategories and total PAQLQ pretest scores while there were highly statistical significant differences between them regarding posttest scores where higher scores were among experimental group. On comparing change in total score, only experimental group showed highly statistically significant improvement (table $4 \& 5)$.

\section{Discussion}

Asthma is considered the third leading cause of hospitalization among children under the age of 15 and one of the prominent causes of absenteeism from school. ${ }^{17}$

To our knowledge, it is the first study in Egypt aimed to evaluate the role of patient centered care in improving outcome of children with asthma in terms of symptom control, pulmonary function test and QoL.

As regard adherence to asthma medication, it was demonstrated that $82.9 \%$ and $75.6 \%$ in experimental and control groups respectively were low adherent to asthma medication at the start of the study. This is mainly due to poor knowledge about asthma and fear of caregivers from side effects of drugs.

Medication adherence was poor in patients with asthma ranging from 30-70\%.6, 18-20 Within experimental group, there was a highly significant improvement in patients' adherence to treatment where $51.2 \%$ of experimental group were highly adherent. However, most of control group (70.7\%) shown low level of adherence.

Other studies reported that patients educated about asthma were less likely to stop their therapy when symptoms get better and uncontrolled asthma was associated with poor education about asthma and/or medication use. ${ }^{21-23}$

Regarding lung function parameters in experimental group, median percentage of PEF change was 56.9 and 18.6 among experimental and control groups respectively $(\mathrm{p}<0.05)$.

These results were in agreement with Denise et al. ${ }^{(8)}$ and who reported that lung function parameters were improved after asthma education.

These findings were also supported by results of similar study done on adult patients which revealed that there was significant increase in average values of lung function indices $\mathrm{FEV}_{1}, \mathrm{FEV}_{1} / \mathrm{FVC}$, PEF among experimental group when compared with values of first session. A 
similar finding was obtained in the control group except from average $\mathrm{FEV}_{1} / \mathrm{FVC}$. In the last session experimental group had significantly higher values than control group. $^{24}$

In contrast with ours, previous studies revealed that there was no significant effect of asthma education on pulmonary function indices such as PEF and FEV ${ }_{1}{ }^{25-26}$

Regarding correctness of inhaler technique, the largest proportion of patients showed incorrect inhaler technique $(80.5 \%, 90.2 \%)$ among experimental and control group respectively at first session. Yet, after the intervention, the proportion of patients perform correct inhaler technique increased from $19.5 \%$ to $65.9 \%$ and this improvement was significantly higher than control group. Denise et al. (8) stated that there was a significant improvement of inhaler technique after education in different published studies which concluded that an incorrect inhalation technique identified during the first evaluation was predictive of a favorable response to the educational intervention.

The key issue of asthma management is to train patients face-to-face and to verify the correct inhalation maneuver. ${ }^{27-28}$

Giraud et al. ${ }^{29}$ found that checking and correcting inhaler technique lead to improved asthma control.

Regarding asthma control at the end of this study, it was found that experimental group significantly improved when compared with control group where among experimental group $65.9 \%$ became well controlled asthma and only $4.9 \%$ still had uncontrolled asthma while among control group $12.2 \%$ only were well controlled and $46.3 \%$ were uncontrolled. In various studies, asthma symptoms control was significantly higher than those in control group. ${ }^{24,30-31}$

There was non-significant difference between group subjected to patient education and usual care regarding asthma control in different studies. ${ }^{25,32}$

Regarding QoL of children with asthma and their caregivers before intervention among experimental and control groups, it was found that the scores of PAQLQ were low in the overall score and all its subcategories (activity limitation, symptoms and emotions) scores where limited activities is the most distressing dimension. Furthermore, PACQLQ scores were low in its overall scores and sub dimensions scores (activity limitation and emotions) where emotions of caregivers appears to be most affected.

This approved that asthma puts a serious burden on children's HRQL and their families. Asthma may worsen the ability of children to carry out daily activities, as well as leading to difficulties in communication, school absenteeism and financial burden on the family.

Previous studies, in Egypt, concluded that childhood asthma significantly impairs QoL of the affected children and their primary caregivers. ${ }^{33-36}$

There is general agreement that asthma causes poor QoL. Asthma education increases disease knowledge, medication adherence and enhances skills for prevention and self-management, consequently improves the patients' QoL which is principle goal of asthma management.

QoL was significantly improved among patients within experimental group compared to control group after intervention in agreement with the study by Yilmaz and Çinar. ${ }^{24}$

In former study, the children absenteeism from school or kindergarten in the experimental group after education was significantly less than before education, while in the control group no significant difference was found at the beginning and the end of the study. ${ }^{26}$ 
Various studies proved that asthma care education program was effective in improving parent reported provider communication skills, the number of days affected by asthma symptoms, and asthma health care use. ${ }^{8,25,30-31,37}$

It is worth noting that some variables showed also significant change (improvement in certain QoL domains, asthma control and accuracy of inhaler technique) in control group yet this occurs to a lesser extent than improvement among experimental group. This denotes that classic consultation is not invaluable but patient centered approach is superior to it in all studied domains.

This study had some strength points represented in presence of control group to clarify the impact of our intervention, randomized distribution of included participants between experimental and control groups, conducting tailored patient education covering different aspects according to patients' needs that determined before intervention and evaluating the program by measuring all variables after intervention.

Yet, this study had some limitation including short period of the study and being conducted at one outpatient clinic.

\section{Conclusion}

By the end of this study, we concluded that patient centered approach can improve outcome of children with asthma in levels out of comparison with classic consultation including medication adherence, level of asthma control, pulmonary function test and QoL. We recommended that all patients should receive asthma education routinely with clinical care and the researchers must do similar studies on a larger sample of children and longer duration at different areas of care like inpatient, ED and primary health units.
Conflict of interest: the authors declared no conflict of interest.

\section{References}

1. GINA. Global strategy for Asthma Management and Prevention [Internet]. Global Initative For Asthma. 2018. Available from: www.ginasthma.org

2. Huo Y, Zhang H-Y. Genetic Mechanisms of Asthma and the Implications for Drug Repositioning. Genes (Basel) [Internet]. 2018 May 3 [cited 2018 Jul 26];9(5):237. Available from: http://www.mdpi.com/20734425/9/5/237

3. Kovesi T, Schuh S, Spier S, Bérubé D, Carr $\mathrm{S}$, Watson W, et al. Achieving control of asthma in preschoolers. CMAJ [Internet]. 2010 Mar 9 [cited 2018 sJul 31];182(4):E172-83. Available from:

http://www.ncbi.nlm.nih.gov/pubmed/19933 790

4. Zedan M, Settin A, Farag M, Ezz-elregal M, Osman E, Fouda A. Prevalence of Bronchial Asthma Among Egyptian School. Egypt J Bronchol. 2009;3(2):124-30.

5. Gill PS. Patient Engagement: An investigation at a primary care clinic. Int $\mathrm{J}$ Gen Med 2013;6:85-98.

6. Chetna A. Shamkuwar, Nalini Kumari , Sushant H. Meshram et al., (2015). "Evaluation of Knowledge, Attitude and Medication Adherence among Asthmatics Outpatients in Tertiary Care Teaching Hospital-A Questionnaire Based Study." Journal of Young Pharmacists $8(1)$ : 39-43. http://jyoungpharm.org/article/823.

7. Pinnock H. (2015). "Supported SelfManagement for Asthma." Breathe 11(2): 98109.

http://breathe.ersjournals.com/cgi/doi/10.11 83/20734735.015614.

8. Denise C.B, Pereira R.P. and Dalcin, P.D. (2013). "Effects of an Outpatient Education Program in Patients with Uncontrolled Asthma." Jornal Brasileiro de Pneumologia 39(3): 272-279. doi: 10.1007/s10346-0050027-7.

9. Morisky D. E., Ang A., Krousel-Wood M. et al. (2008). "Predictive Validity of a Medication Adherence Measure in an Outpatient Setting." 
Journal of Clinical Hypertension 10(5): 348$354 . \quad$ https://doi.org/10.1111/j.17517176.2008.07572.x.

10. Janežič Ana, Locatelli Igor and Kos Mitja (2017). "Criterion Validity of 8-Item Morisky Medication Adherence Scale in Patients with Asthma." PLoS ONE 12 (11): e0187835. https://doi.org/10.1371/journal.pone.018783

5 .

11. Juniper E. F., Guyatt G.H., Feeny D.H. et al. (1996a). "Measuring Quality of Life in Children with Asthma." Quality of life research 5(1): 35-46.

12. Juniper E. F., Guyatt G.H., Feeny D.H. et al. (1996b). "Measuring Quality of Life in the Parents Children with Asthma." Quality of life research 5(1): 27-34.

13. National Asthma Council Australia (2016). "Inhaler technique for people with asthma or COPD” .https://assets.nationalasthma. org.au/resources/

Inhaler_technique_for_people_with_asthma_o r_COPD.pdf.

14. Ostrowski S. and Barud W. (2006). "Factors Influencing Lung Function: Are the Predicted Values for Spirometry Reliable Enough?" Journal of Physiology and Pharmacology 57(4): 263-271.

15. GINA (Global Initiative For Asthma) (2017). "Global Strategy For Asthma Management and Prevention." http://ginasthma.org/2017-gina-report-globalstrat (September 16, 2017).

16. El-Gilany A., El-Wehady A. and El-Wasify M. (2012). "Updating and Validation of the Socioeconomic Status Scale for Health Research in Egypt." Eastern Mediterranean health journal 18(9): 962-68. doi: 10.26719/2012.18.9.962.

17. Pearson W. S., Goates S. A., Harrykissoon S. D. et al. (2014). "State-Based Medicaid Costs for Pediatric Asthma Emergency Department Visits." Preventing Chronic Disease 11: E108. doi: 10.5888/pcd11.140139 (August 10, 2018). 18. Gaude G. S., Hattiholi J. and Chaudhury A. (2014). "Role of Health Education and SelfAction Plan in Improving the Drug Compliance in Bronchial Asthma." Journal of Family Medicine and Primary Care 3(1): 33. http://www.ncbi.nlm.nih.gov/pubmed/2479123 4 (October 4, 2018).
19. Marchioro J., Gazzotti M.R., Nascimento O.A. et al. (2014). "Level of Asthma Control and Its Relationship with Medication Use in Asthma Patients in Brazil." Jornal brasileiro de pneumologia 40(5): 487-494. http://dx.doi.org/10.1590/S1806-

37132014000500004 (October 4, 2018).

20. Clayton Sadie (2014). "Adherence to Asthma Medication.” Nurse Prescribing 12(2): 68-74.

https://doi.org/10.12968/npre.2014.12.2.68

(August 3, 2018).

21. Bateman E. D., Hurd S.S., Barnes P.J. et al. (2008). "Global Strategy for Asthma Management and Prevention: GINA Executive Summary." European Respiratory Journal 31(1): 143-178. http://www.ncbi.nlm.nih.gov/ pubmed/ 18166595 (September 30, 2018).

22. Hinchageri S. S., Patil N., Karan K. et al. (2012). "Assessment of Medication Adherence and Factors Affecting to Mediaction Adherence in Asthma Patients by Clinical Pharmacist." International Research Journal of Pharmacy 3(3): 211-15. https://irjponline.com/ admin/php/uploads/936_pdf.pdf (October 4, 2018).

23. Al-Muhsen S., Horanieh N., Dulgom S. et al. (2015). "Poor Asthma Education and Medication Compliance Are Associated with Increased Emergency Department Visits by Asthmatic Children." Annals of Thoracic Medicine 10(2):123-131. https://dx.doi.org/ 10.4103\%2F1817-1737.150735 (June10, 2018).

24. Yilmaz F. T. and Çinar S. (2015). "Effect of Educational on Symptom Control and Quality of Life on Asthmatic Patients." Anatolian Journal of Clinical Investigation 9(2): 47-54. 25. Pbert Lori, Madison J.M., Druker S. et al. (2012). "Effect of Mindfulness Training on Asthma Quality of Life and Lung Function: A Randomised Controlled Trial.” Thorax 67(9): 769-776. http://www.ncbi.nlm.nih.gov/ pubmed/22544892 (September 29, 2018).

26. Kalantari A. , Movahedi M., Moin M. et al. (2017). "Effect of Education on Asthma Control in Children: A Quasi-Experimental Study." International Journal of Pediatrics, 5(12), pp.6421-6429.doi: 10.22038/ijp. 2017.26728.2302 (September 29, 2018). 
27. Aydemir Yusuf (2015). "Assessment of the Factors Affecting the Failure to Use Inhaler Devices before and after Training." Respiratory Medicine 109(4): 451-458. doi: 10.1016/j.rmed.2015.02.011 (December 7, 2018).

28. Scichilone N. (2015). "Asthma Control: The Right Inhaler for the Right Patient." Advances in therapy 32(4): 285-292. https://doi.org/10.1007/s12325-015-0201-9 (October 7, 2018).

29. Giraud V., Allaert F. A. and Roche N. (2011). "Inhaler Technique and Asthma: Feasability and Acceptability of Training by Pharmacists." Respiratory Medicine 105(12): 1815-1822. https://www.sciencedirect.com/ science/article /pii/S0954611111002186 (August 3, 2018).

30. Iio M., Takenaka K., Narita M. et al. (2014). "Development and Effectiveness of a Tailored Education Program for Caregivers of Asthmatic Children." Arerugi $=$ [Allergy $]$ 63(2): 187-203. http://www.ncbi.nlm.nih.gov/pubmed/2471418 4 (September 29, 2018).

31. Sun H.W., Wang J.P., Wang S.Z. et al. (2010). "Effect of Educational and Psychological Intervention on the Quality of Life of Asthmatic Patients." Respiratory care 55(6): 725-728. http://www.ncbi.nlm.nih.gov/ pubmed/20507655 (October 3, 2018).

32. Arıkan-Ayyıldız Z., Isik S., ÇaglayanSözmen S. et al. (2016). "Efficacy of Asthma Education Program on Asthma Control in Children with Uncontrolled Asthma." The Turkish journal of pediatrics 58(4): 383-388. http://www.turkishjournalpediatrics.org/upload s/pdf_TJP_1615.pdf (October 7, 2018).
33. Cabana M. D. ,Slish K.K., Evans D. et al. (2014). "Impact of Physician Asthma Care Education on Patient Outcomes." Health Education \& Behavior 41(5): 509-517. http://journals.sagepub.com/doi/10.1177/1090 198114547510 (September 29, 2018).

34. ElGendi S. D., Mostaf, S.A., Walli M.H. et al. (2017). "Assessment of Health-Related Quality of Life in Asthmatic Children and Their Caregivers." International Journal of Medical Science and Public Health 6(4): 798-806. doi: $\quad$ 10.5455/ijmsph.2017.0638206122016 (October 5, 2018).

35. Elshazly H., El Mahalawy I., Gabr H. et al. (2015). "Quality of Life among Asthmatic Children Attending the Outpatient Clinic in Menoufia University Hospital." Menoufia $\begin{array}{lll}\text { Medical Journal 28(2): } 442 . & .4\end{array}$ http://www.mmj.eg.net/

text.asp?2015/28/2/442/163899 (July 30, 2018).

36. Eissa M. M., Elderiny G. F. and Elbanna A. G. (2017). "Evaluation of Health-Related Quality of Life among Children Attending Asthma Clinic in Alexandria University Children ' s Hospital." journal of medical science and clinical research 05(06): 23093 23102. https://dx.doi.org/ 10.18535 /jmscr /v5i6.49 (October 19,2017).

37. Cabana M. D. ,Slish K.K., Evans D. et al. (2014). "Impact of Physician Asthma Care Education on Patient Outcomes." Health Education \& Behavior 41(5): 509-517. http://journals.sagepub.com/doi/10.1177/1090 198114547510 (September 29, 2018). 